\title{
QUASI-MODAL OPERATORS ON DISTRIBUTIVE NEARLATTICES
}

\author{
ISMAEL CALOMINO, SERGIO A. CELANI, AND LUCIANO J. GONZÁLEZ
}

\begin{abstract}
We introduce the notion of quasi-modal operator in the variety of distributive nearlattices, which turns out to be a generalization of the necessity modal operator studied in [S. Celani and I. Calomino, Math. Slovaca 69 (2019), no. 1, 35-52]. We show that there is a one to one correspondence between a particular class of quasi-modal operators on a distributive nearlattice and the class of possibility modal operators on the distributive lattice of its finitely generated filters. Finally, we consider the concept of quasi-modal congruence, and we show that the lattice of quasi-modal congruences of a quasi-modal distributive nearlattice is isomorphic to the lattice of congruences of the lattice of finitely generated filters with a possibility modal operator.
\end{abstract}

\section{INTRODUCTION AND PRELIMINARIES}

Implication algebras, also called Tarski algebras, were introduced and studied by Abbott in [1, 2]. A natural generalization of implication algebras is the class of nearlattices: join-semilattices with greatest element in which every principal filter is a bounded lattice. These structures were studied by different authors in 17, 21, 20, 12, 14, 15, 8, 9, 18, 19. A particular class of nearlattices is the class of distributive nearlattices, i.e., join-semilattices with greatest element in which every principal filter is a bounded distributive lattice. Clearly, every bounded distributive lattice is in particular a distributive nearlattice. Thus, distributive nearlattices are generalizations both of implication algebras and distributive lattices. In [8] a full duality is developed for distributive nearlattices and in [9] some applications are given. Recently, distributive nearlattices were studied from the point of view of algebraic logic. In [18, 19] a sentential logic was defined and studied in such a way that its algebraic counterpart is the class of distributive nearlattices.

In the class of distributive nearlattices, a notion of necessity modal operator was introduced and studied (see [10]) as a generalization of necessity modal operators on bounded distributive lattices $([16,22,11])$ and modal operators on Tarski algebras ([7]). On the other hand, there is a strong connection between a nearlattice and its filters. To be more precise, Hickman and Cornish prove in [17] that a nearlattice is

2010 Mathematics Subject Classification. Primary 06A12; Secondary 03G10, 06D75.

Key words and phrases. Nearlattice; modal operator; filter; finitely generated filter.

This research was supported by CONICET under grant PIP 112-201501-00412. 
distributive if and only if the lattice of its finitely generated filters is distributive. Motivated by this fact, we ask ourselves which operators on a distributive nearlattice should correspond to the modal operators on the distributive lattice of its finitely generated filters. Thus, we introduce the so-called quasi-modal operators in the class of distributive nearlattices, and we show that these are a generalization of the necessity modal operators given in [10]. The main aim of this article is to study quasi-operators on distributive nearlattices, and prove that they are in one to one correspondence with possibility modal operators on the distributive lattice of its finitely generated filters. We define the notion of quasi-congruence in quasi-modal distributive nearlattices and we give a representation theorem.

The paper is organized as follows. In the remaining part of this section we review some definitions. In Section 2 we define quasi-modal operators and finite quasi-modal operators on distributive nearlattices. We show a correspondence between finite quasi-modal operators on a distributive nearlattice $\mathbf{A}$ and possibility modal operators on the distributive lattice of its finitely generated filters $\operatorname{Fi}_{\mathrm{f}}(\mathbf{A})$. We also give some characterizations and a representation theorem. In Section 3 we introduce the notion of qm-congruence, i.e., equivalence relations compatible with the quasi-modal operator. We prove that there is an isomorphism between qm-congruences of a quasi-modal distributive nearlattice and congruences of distributive lattice of its finitely generated filters with a possibility modal operator.

Let $\mathbf{A}=\langle A, \vee, 1\rangle$ be a join-semilattice with greatest element. A filter is a subset $F$ of $A$ such that $1 \in F$, if $a \leq b$ and $a \in F$ then $b \in F$, and if $a, b \in F$ then $a \wedge b \in F$, whenever $a \wedge b$ exists. If $X$ is a subset of $A$, the least filter containing $X$ is called the filter generated by $X$ and will be denoted by $\operatorname{Fig}(X)$. A filter $G$ is said to be finitely generated if $G=\operatorname{Fig}(X)$, for some finite subset $X$ of $A$. If $X=\{a\}$, then $\operatorname{Fig}(\{a\})=[a)=\{x \in A: a \leq x\}$, called the principal filter of $a$. We denote by $\operatorname{Fi}(A)$ and $\operatorname{Fi}_{\mathrm{f}}(A)$ the sets of all filters and of all finitely generated filters of $A$, respectively. A subset $I$ of $A$ is called an ideal if $a \leq b$ and $b \in I$ implies $a \in I$, and $a, b \in I$ implies $a \vee b \in I$. If $X$ is a non-empty set, the least ideal containing $X$ is called the ideal generated by $X$ and will be denoted by $\operatorname{Idg}(X)$. We shall say that a non-empty proper ideal $P$ is prime if for all $a, b \in A, a \wedge b \in I$ implies $a \in I$ or $b \in I$, whenever $a \wedge b$ exists. We denote by $\operatorname{Id}(A)$ and $\mathrm{X}(A)$ the sets of all ideals and of all prime ideals of $A$, respectively.

Definition 1.1. Let $\mathbf{A}$ be a join-semilattice with greatest element. We say that $\mathbf{A}$ is a nearlattice if each principal filter is a bounded lattice.

Let $\mathbf{A}$ be a nearlattice. For each $a \in A$, we will denote the meet operation of the lattice $[a)$ by $\wedge_{a}$. Thus, $\left\langle[a), \vee, \wedge_{a}, a, 1\right\rangle$ is a bounded lattice. Note that if $x, y \in[a)$ and $b \leq a$, then $x, y \in[b)$ and $x \wedge_{a} y=x \wedge_{b} y$. The nearlattices form a variety. This fact was first proved in [21] and independently in [15]. In [3] the authors found a smaller equational base.

Theorem $1.2([3])$. Let $\mathbf{A}$ be a nearlattice. Let $m: A^{3} \rightarrow A$ be the ternary operation given by $m(x, y, z)=(x \vee z) \wedge_{z}(y \vee z)$. The following identities are satisfied: 
(1) $m(x, y, x)=x$.

(2) $m(m(x, y, z), m(y, m(u, x, z), z), w)=m(w, w, m(y, m(x, u, z), z))$.

(3) $m(x, x, 1)=1$.

Conversely, let $\mathbf{A}=\langle A, m, 1\rangle$ be an algebra of type $(3,0)$ satisfying the identities (1)-(3). If we define $x \vee y=m(x, x, y)$, then $\mathbf{A}$ is a join-semilattice with greatest element. Moreover, for each $a \in A,[a]$ is a bounded lattice where for $x, y \in[a)$, $x \wedge_{a} y=m(x, y, a)$. Hence, $\mathbf{A}$ is a nearlattice.

For each nearlattice $\mathbf{A}, m$ will denote the operation defined in Theorem 1.2 We introduce the following notation. For each natural number $n$ we define inductively, for every $a_{1}, \ldots, a_{n}, b \in A$, the element $m^{n-1}\left(a_{1}, \ldots, a_{n}, b\right)$ as follows:

- $m^{0}\left(a_{1}, b\right)=m\left(a_{1}, a_{1}, b\right)$;

- for $n>1, m^{n-1}\left(a_{1}, \ldots, a_{n}, b\right)=m\left(m^{n-2}\left(a_{1}, \ldots, a_{n-1}, b\right), a_{n}, b\right)$.

So, $m^{n-1}\left(a_{1}, \ldots, a_{n}, b\right)=\left(a_{1} \vee b\right) \wedge_{b} \ldots \wedge_{b}\left(a_{n} \vee b\right)$, and, in particular, $m^{0}\left(a_{1}, b\right)=$ $a_{1} \vee b$ and $m^{1}\left(a_{1}, a_{2}, b\right)=m\left(a_{1}, a_{2}, b\right)$, where $m\left(a_{1}, a_{2}, b\right)$ is given by Theorem 1.2

Definition 1.3. Let $\mathbf{A}$ be a nearlattice. We say that $\mathbf{A}$ is distributive if each principal filter is a bounded distributive lattice.

Example 1.4 ([1, 2]). If $\mathbf{A}=\langle A, \rightarrow, 1\rangle$ is an implication algebra, then it is known that $\mathbf{A}=\langle A, \vee, 1\rangle$, where $x \vee y=(x \rightarrow y) \rightarrow y$ is a join-semilattice with greatest element such that each principal filter is a Boolean algebra. Hence, every implication algebra is in particular a distributive nearlattice.

Let $\mathbf{A}$ be a nearlattice. Consider the latttice $\operatorname{Fi}(\mathbf{A})=\langle\operatorname{Fi}(A), \underline{\vee}, \bar{\wedge},\{1\}, A\rangle$, where the least element is $\{1\}$, the greatest element is $A, G \bar{\wedge} H=G \cap H$, and $G \underline{\vee} H=\operatorname{Fig}(G \cup H)$, for every $G, H \in \operatorname{Fi}(A)$. On the other hand, from the results given in [17, we have the following characterization of the filter generated by a subset $X$ of $A$ :

$$
\operatorname{Fig}(X)=\left\{a \in A: \exists x_{1}, \ldots, x_{n} \in[X)\left(a=x_{1} \wedge \cdots \wedge x_{n}\right)\right\} .
$$

In particular, if $X=\left\{a_{1}, \ldots, a_{n}\right\}$, then

$$
\operatorname{Fig}(X)=\left[a_{1}\right) \underline{\vee} \ldots \underline{\vee}\left[a_{n}\right)=\left\{a \in A: a=m^{n-1}\left(a_{1}, \ldots, a_{n}, a\right)\right\} .
$$

So, the structure $\operatorname{Fi}_{\mathrm{f}}(\mathbf{A})=\left\langle\operatorname{Fi}_{\mathrm{f}}(A), \underline{\vee}\right\rangle$ is a sub join-semilattice of $\operatorname{Fi}(\mathbf{A})$.

Theorem 1.5 ([17, 13]). Let A be a nearlattice. The following conditions are equivalent:

(1) A is distributive.

(2) $\operatorname{Fi}(\mathbf{A})$ is a bounded distributive lattice.

(3) $\mathrm{Fi}_{\mathrm{f}}(\mathbf{A})$ is a bounded distributive lattice.

Theorem $1.6([20])$. Let $\mathbf{A}$ be a distributive nearlattice. Let $I \in \operatorname{Id}(A)$ and let $F \in \operatorname{Fi}(A)$ be such that $I \cap F=\emptyset$. Then there exists $P \in \mathrm{X}(A)$ such that $I \subseteq P$ and $P \cap F=\emptyset$.

If $\mathbf{A}$ and $\mathbf{B}$ are two distributive nearlattices, then a map $h: A \rightarrow B$ is a homomorphism if $h(1)=1, h(a \vee b)=h(a) \vee h(b)$, and $h(a \wedge b)=h(a) \wedge h(b)$, whenever $a \wedge b$ 
exists. In other words, $h$ is a homomorphism if $h(m(a, b, c))=m(h(a), h(b), h(c))$, for every $a, b, c \in A$. Note that if $a, b \in A$ such that $a \wedge b$ exists, then $h(a) \wedge h(b)$ exists in $B$. We have the following representation theorem.

Theorem 1.7 ([20]). Let $\mathbf{A}$ be a distributive nearlattice and $\varphi_{A}: A \rightarrow \mathcal{P}_{d}(\mathrm{X}(A))$ the map given by $\varphi_{A}(a)=\{P \in \mathrm{X}(A): a \notin P\}$. Then $\varphi_{A}$ is an embedding of $\mathbf{A}$ into $\mathcal{P}_{d}(\mathrm{X}(A))$. Hence, $\mathbf{A}$ is isomorphic to the subalgebra $\varphi_{A}[\mathbf{A}]=\left\{\varphi_{A}(a): a \in A\right\}$.

\section{Quasi-Modal Distributive NEARLATtiCES}

In [5, 6], the author studies the class of quasi-modal algebras as a generalization of modal algebras. A quasi-modal algebra is a Boolean algebra with a map between the algebra and the set of its ideals. This kind of maps are not operations in the algebra, and the quasi-modal algebras are not algebras according to the standard terminology of universal algebra, but they have some properties similar to those of modal operators. Following this line of research, in this section we study quasi-modal operators on distributive nearlattices and their connection with modal operators on the distributive lattice of its finitely generated filters.

\subsection{Quasi-modal operators.}

Definition 2.1. Let $\mathbf{A}$ be a distributive nearlattice. A quasi-modal operator defined on $\mathbf{A}$ is a map $\nabla: A \rightarrow \operatorname{Fi}(A)$ that satisfies the following conditions:

(1) $\nabla 1=\{1\}$

(2) $\nabla(a \wedge b)=\nabla a \underline{\vee} \nabla b$, whenever $a \wedge b$ exists.

A finite quasi-modal operator defined on $\mathbf{A}$ is a quasi-modal operator that satisfies $\nabla a \in \mathrm{Fi}_{\mathrm{f}}(A)$, for every $a \in A$. A pair $\langle\mathbf{A}, \nabla\rangle$ is a quasi-modal distributive nearlattice, or simply qm-distributive nearlattice, if $\mathbf{A}$ is a distributive nearlattice and $\nabla$ is a quasi-modal operator defined on $\mathbf{A}$. Analogously, a pair $\langle\mathbf{A}, \nabla\rangle$ is a finite quasi-modal distributive nearlattice, or simply fqm-distributive nearlattice, if $\mathbf{A}$ is a distributive nearlattice and $\nabla$ is a finite quasi-modal operator defined on $\mathbf{A}$.

Let us denote by $\mathcal{D} \mathcal{N}_{\nabla}$ and $\mathcal{D} \mathcal{N}_{\nabla \text { f }}$ the classes of qm-distributive nearlattices and fqm-distributive nearlattices, respectively. The classes $\mathcal{D} \mathcal{N}_{\nabla}$ and $\mathcal{D} \mathcal{N}_{\nabla \text { f }}$ are not varieties, because $\nabla$ is not an operation on $\mathbf{A}$. On the other hand, note that $\nabla$ is a map that reverses the order.

Example 2.2. Let $\mathbf{A}$ be a distributive nearlattice. If we define $\nabla: A \rightarrow \mathrm{Fi}_{\mathrm{f}}(A)$ by $\nabla a=[a)$, for every $a \in A$, then the structure $\langle\mathbf{A}, \nabla\rangle$ is a fqm-distributive nearlattice.

Example 2.3. In [10, a necessity modal operator on a distributive nearlattice $\mathbf{A}$ was introduced as a map $\square: A \rightarrow A$ such that $\square 1=1$ and $\square(a \wedge b)=\square a \wedge \square b$, whenever $a \wedge b$ exists. It is easy to see that $\square$ induces a finite quasi-modal operator $\nabla_{\square}$ if for each $a \in A$ we put $\nabla_{\square}(a)=[\square a)$. Conversely, if $\langle\mathbf{A}, \nabla\rangle$ is a fqm-distributive nearlattice such that for each $a \in A$ the filter $\nabla a$ is principal, then the map $\square_{\nabla}: A \rightarrow A$ given by $\square_{\nabla}(a)=b$ if and only if $\nabla a=[b)$, defines a necessity modal operator on $\mathbf{A}$. Therefore, finite quasi-modal operators are a natural generalization of necessity modal operators in the class of distributive nearlattices. 
Example 2.4. Let $X$ be a set and $R \subseteq X \times X$. Consider the distributive nearlattice $\langle\mathcal{P}(X), \cup, X\rangle$ and take the map $\nabla_{R}: \mathcal{P}(X) \rightarrow \operatorname{Fi}(\mathcal{P}(X))$ defined by

$$
\nabla_{R}(U)=\left\{V \in \mathcal{P}(X): \square_{R}(U) \subseteq V\right\},
$$

where $U \in \mathcal{P}(X)$ and

$$
\square_{R}(U)=\{x \in X: R(x) \subseteq U\},
$$

i.e., $\nabla_{R}(U)=\left[\square_{R}(U)\right)$. It follows that $\nabla_{R}$ is a quasi-modal operator on $\mathcal{P}(X)$. Indeed, since $\square_{R}(X)=X$, we have $\nabla_{R}(X)=\{X\}$. If $W \in \nabla_{R}(U \cap V)$, then $\square_{R}(U \cap V)=\square_{R}(U) \cap \square_{R}(V) \subseteq W$ and

$$
W=\left(\square_{R}(U) \cup W\right) \cap\left(\square_{R}(V) \cup W\right),
$$

where $\square_{R}(U) \cup W \in \nabla_{R}(U)$ and $\square_{R}(V) \cup W \in \nabla_{R}(V)$. So, $W \in \nabla_{R}(U) \underline{\vee} \nabla_{R}(V)$ and $\nabla_{R}(U \cap V) \subseteq \nabla_{R}(U) \underline{\vee} \nabla_{R}(V)$. On the other hand, since $\nabla_{R}$ inverts the order, we have $\nabla_{R}(U) \underline{\vee} \nabla_{R}(V) \subseteq \nabla_{R}(U \cap V)$. Then $\nabla_{R}(U \cap V)=\nabla_{R}(U) \underline{\vee} \nabla_{R}(V)$ and $\left\langle\mathcal{P}(X), \nabla_{R}\right\rangle$ is a qm-distributive nearlattice.

Let $\langle\mathbf{A}, \nabla\rangle \in \mathcal{D} \mathcal{N}_{\nabla}$. Let $D$ be a subset of $A$ and

$$
\gamma(D)=\{a \in A: \nabla a \cap D=\emptyset\} .
$$

Theorem 2.5. Let $\mathbf{A}$ be a distributive nearlattice and $\nabla: A \rightarrow \operatorname{Fi}(A)$ a map. The following conditions are equivalent:

(1) $\nabla$ is a quasi-modal operator on $\mathbf{A}$.

(2) $\nabla$ inverts the order and $\gamma(P) \in \mathrm{Fi}(A)$, for every $P \in \mathrm{X}(A)$.

Proof. $(1) \Rightarrow(2) \quad$ Let $P \in \mathrm{X}(A)$. As $\nabla 1=\{1\}$ and $P$ is a proper ideal, $\nabla 1 \cap P=\emptyset$ and $1 \in \gamma(P)$. Let $a, b \in A$ be such that $a \leq b$ and $a \in \gamma(P)$. Since $\nabla$ inverts the order, $\nabla b \subseteq \nabla a$ and $\nabla a \cap P=\emptyset$. So, $\nabla b \cap P=\emptyset$ and $b \in \gamma(P)$. If $a, b \in \gamma(P)$ such that $a \wedge b$ exists, then $\nabla a \cap P=\emptyset$ and $\nabla b \cap P=\emptyset$. Suppose that $\nabla(a \wedge b) \cap P \neq \emptyset$, i.e., there is $x \in A$ such that $x \in(\nabla a \underline{\vee} \nabla b) \cap P \neq \emptyset$. Thus, there exist $x_{1}, \ldots, x_{n} \in$ $\nabla a \cup \nabla b$ such that $x_{1} \wedge \ldots \wedge x_{n}$ exists and $x=x_{1} \wedge \ldots \wedge x_{n}$. Since $x \in P$ and $P$ is prime, there exists $i \in\{1, \ldots, n\}$ such that $x_{i} \in P$. It follows that $x_{i} \in \nabla a \cap P$ or $x_{i} \in \nabla b \cap P$, which is a contradiction. Then $\nabla(a \wedge b) \cap P=\emptyset$ and $a \wedge b \in \gamma(P)$. Therefore, $\gamma(P) \in \mathrm{Fi}(A)$, for every $P \in \mathrm{X}(A)$.

$(2) \Rightarrow(1)$ If $\nabla 1 \neq\{1\}$, then there is $x \in A$ such that $x \neq 1$ and $x \in \nabla 1$. So, by Theorem 1.6. there exists $P \in \mathrm{X}(A)$ such that $x \in P$. It follows that $x \in \nabla 1 \cap P$ and $1 \notin \gamma(P)$, which is a contradiction because $\gamma(P)$ is a filter. Then $\nabla 1=\{1\}$. Let $a, b \in A$ be such that $a \wedge b$ exists. Since $\nabla$ inverts the order, $\nabla a \subseteq \nabla(a \wedge b)$ and $\nabla b \subseteq \nabla(a \wedge b)$. So, $\nabla a \underline{\vee} \nabla b \subseteq \nabla(a \wedge b)$. We show the other inclusion. Suppose there is $x \in \nabla(a \wedge b)$ such that $x \notin \nabla a \vee \nabla b$. By Theorem 1.6. there exists $P \in \mathrm{X}(A)$ such that $x \in P$ and $P \cap(\nabla a \underline{\vee} \nabla b)=\emptyset$. Then $\nabla a \cap P=\emptyset$ and $\nabla b \cap P=\emptyset$, i.e., $a, b \in \gamma(P)$. As $\gamma(P)$ is a filter, $a \wedge b \in \gamma(P)$ and $\nabla(a \wedge b) \cap P=\emptyset$. On the other hand, $x \in \nabla(a \wedge b) \cap P$ and $a \wedge b \notin \gamma(P)$, which is a contradiction. So, $\nabla(a \wedge b) \subseteq \nabla a \underline{\vee} \nabla b$ and $\nabla$ is a quasi-modal operator on $\mathbf{A}$.

Proposition 2.6. Let $\langle\mathbf{A}, \nabla\rangle \in \mathcal{D N}_{\nabla}$. Let $a \in A$ and $P \in \mathrm{X}(A)$. Then $\nabla a \cap P \neq \emptyset$ if and only if there exists $Q \in \mathrm{X}(A)$ such that $\gamma(P) \cap Q=\emptyset$ and $a \in Q$. 
Proof. If $\nabla a \cap P \neq \emptyset$, then $a \notin \gamma(P)$. So, by Theorems 2.5 and 1.6 , there exists $Q \in \mathrm{X}(A)$ such that $\gamma(P) \cap Q=\emptyset$ and $a \in Q$. The reciprocal is immediate.

2.2. Finite quasi-modal operators. Now, we will study the relationship between finite quasi-modal operators on a distributive nearlattice $\mathbf{A}$ and possibility modal operators on the bounded distributive lattice $\mathrm{Fi}_{\mathrm{f}}(\mathbf{A})$. Recall that a possibility modal operator on a bounded distributive lattice $\mathbf{L}=\langle L, \vee, \wedge, 0,1\rangle$ is a map $\diamond: L \rightarrow L$ such that $\diamond 0=0$ and $\diamond(a \vee b)=\diamond a \vee \diamond b$, for every $a, b \in L$.

Definition 2.7. Let $\langle\mathbf{A}, \nabla\rangle \in \mathcal{D} \mathcal{N}_{\nabla}$. For a subset $X \subseteq A$, we define

$$
\diamond_{\nabla}(X)=\operatorname{Fig}(\bigcup\{\nabla x: x \in X\}) \text {. }
$$

Lemma 2.8. Let $\langle\mathbf{A}, \nabla\rangle \in \mathcal{D} \mathcal{N}_{\nabla}$. Then $\diamond_{\nabla}([a))=\nabla a$, for every $a \in A$.

Proof. Let $y \in \nabla_{\nabla}([a))=$ Fig $(\bigcup\{\nabla x: x \in[a)\})$. Then there are $y_{1}, \ldots, y_{n} \in$ $\bigcup\{\nabla x: x \in[a)\}$ such that $y_{1} \wedge \ldots \wedge y_{n}$ exists and $y=y_{1} \wedge \ldots \wedge y_{n}$. So, there exist $x_{1}, \ldots, x_{n} \in A$ such that $a \leq x_{i}$ and $y_{i} \in \nabla x_{i}$, for all $i \in\{1, \ldots, n\}$. Then $\nabla x_{i} \subseteq$ $\nabla a$ and $y_{i} \in \nabla a$, for all $i \in\{1, \ldots, n\}$. Since $\nabla a$ is a filter, $y_{1} \wedge \ldots \wedge y_{n}=y \in \nabla a$. The other inclusion is easy to follow.

Proposition 2.9. Let $\langle\mathbf{A}, \nabla\rangle \in \mathcal{D} \mathcal{N}_{\nabla \mathrm{f}}$. Then the map $\diamond_{\nabla}: \mathrm{Fi}_{\mathrm{f}}(A) \rightarrow \mathrm{Fi}_{\mathrm{f}}(A)$ given by $\square$ is a possibility modal operator on $\mathrm{Fi}_{\mathrm{f}}(\mathbf{A})$, i.e., $\nabla_{\nabla}$ satisfies the following conditions:

(1) $\diamond_{\nabla}([1))=[1)$.

(2) $\diamond_{\nabla}(F \underline{\vee} G)=\diamond_{\nabla}(F) \underline{\vee} \diamond_{\nabla}(G)$, for every $F, G \in \mathrm{Fi}_{\mathrm{f}}(A)$.

Proof. We prove that $\nabla_{\nabla}$ is well-defined. If $F \in \mathrm{Fi}_{\mathrm{f}}(A)$, there exist $a_{1}, \ldots, a_{n} \in A$ such that $F=\left[a_{1}\right) \underline{\vee} \ldots \underline{\vee}\left[a_{n}\right)$. Let us show that $\diamond_{\nabla}(F)=\diamond_{\nabla}\left(\left[a_{1}\right)\right) \underline{\vee} \ldots \underline{\vee} \diamond_{\nabla}\left(\left[a_{n}\right)\right)$. If $y \in \diamond_{\nabla}(F)$, then there are $y_{1}, \ldots, y_{m} \in$ Fig $(\bigcup\{\nabla x: x \in F\})$ such that $y_{1} \wedge \ldots \wedge y_{m}$ exists and $y=y_{1} \wedge \ldots \wedge y_{m}$. So, there exist $x_{1}, \ldots, x_{m} \in F$ such that $y_{i} \in \nabla x_{i}$, for all $i \in\{1, \ldots, m\}$. As $x_{i} \in F=\left[a_{1}\right) \underline{\vee} \ldots \underline{\vee}\left[a_{n}\right)$, we have $x_{i}=\left(a_{1} \vee x_{i}\right) \wedge_{x_{i}} \ldots \wedge_{x_{i}}\left(a_{n} \vee x_{i}\right)$, for all $i \in\{1, \ldots, m\}$. It follows that

$$
\nabla x_{i}=\nabla\left(a_{1} \vee x_{i}\right) \underline{\vee} \ldots \underline{\vee} \nabla\left(a_{n} \vee x_{i}\right),
$$

for all $i \in\{1, \ldots, m\}$. Since $a_{j} \leq a_{j} \vee x_{i}$, we have $\nabla\left(a_{j} \vee x_{i}\right) \subseteq \nabla\left(a_{j}\right)$, for all $j \in\{1, \ldots, n\}$. Hence,

$$
\nabla\left(a_{1} \vee x_{i}\right) \underline{\vee} \ldots \underline{\vee} \nabla\left(a_{n} \vee x_{i}\right) \subseteq \nabla a_{1} \underline{\vee} \ldots \underline{\vee} \nabla a_{n}
$$

for all $i \in\{1, \ldots, m\}$. So, $y_{i} \in \nabla x_{i} \subseteq \nabla a_{1} \underline{\vee} \ldots \underline{\vee} \nabla a_{n}$, for all $i \in\{1, \ldots, m\}$ and $y \in \nabla a_{1} \underline{\vee} \ldots \underline{\vee} \nabla a_{n}$. Thus, by Lemma 2.8, $\nabla_{\nabla}(F) \subseteq \nabla_{\nabla}\left(\left[a_{1}\right)\right) \underline{\vee} \ldots \underline{\vee} \nabla_{\nabla}\left(\left[a_{n}\right)\right)$. The other inclusion is immediate.

By definition, $\nabla_{\nabla}([1))=[1)$. Finally, we consider $F, G \in \mathrm{Fi}_{\mathrm{f}}(A)$. Then there exist $a_{1}, \ldots, a_{n}, b_{1}, \ldots, b_{m} \in A$ such that $F=\left[a_{1}\right) \underline{\vee} . . \underline{\vee}\left[a_{n}\right)$ and $G=\left[b_{1}\right) \underline{\vee} . . \underline{\vee}\left[b_{m}\right)$. Thus,

$\diamond_{\nabla}(F \underline{\vee} G)=\diamond_{\nabla}\left(\left[a_{1}\right)\right) \underline{\vee} \ldots \underline{\vee} \diamond_{\nabla}\left(\left[a_{n}\right)\right) \underline{\vee} \diamond_{\nabla}\left(\left[b_{1}\right)\right) \underline{\vee} \ldots \underline{\vee} \diamond_{\nabla}\left(\left[b_{m}\right)\right)=\diamond_{\nabla}(F) \underline{\vee} \diamond_{\nabla}(G)$.

Therefore, $\diamond_{\nabla}$ is a possibility modal operator on $\operatorname{Fi}_{\mathrm{f}}(\mathbf{A})$. 
Proposition 2.10. Let $\mathbf{A}$ be a distributive nearlattice. If $\diamond: \operatorname{Fi}_{\mathrm{f}}(A) \rightarrow \operatorname{Fi}_{\mathrm{f}}(A)$ is a possibility modal operator on $\mathrm{Fi}_{\mathrm{f}}(\mathbf{A})$, then the map $\nabla_{\diamond}: A \rightarrow \mathrm{Fi}_{\mathrm{f}}(A)$ given by $\nabla_{\diamond} a=\diamond([a))$ is a finite quasi-modal operator on $\mathbf{A}$.

Proof. It is clear that $\nabla_{\diamond} 1=\{1\}$. Let $a, b \in A$ be such that $a \wedge b$ exists. Then

$$
\nabla_{\diamond}(a \wedge b)=\diamond([a \wedge b))=\diamond([a) \underline{\vee}[b))=\diamond([a)) \underline{\vee} \diamond([b))=\nabla_{\diamond}(a) \underline{\vee} \nabla_{\diamond}(b),
$$

and $\nabla_{\diamond}$ is a finite quasi-modal operator on $\mathbf{A}$.

Theorem 2.11. Let $\mathbf{A}$ be a distributive nearlattice. The following properties are satisfied:

(1) If $\nabla$ is a finite quasi-modal operator on $\mathbf{A}$, then $\nabla=\nabla_{\diamond_{\nabla}}$.

(2) If $\diamond$ is a possibility modal operator on $\mathrm{Fi}_{\mathrm{f}}(\mathbf{A})$, then $\diamond=\diamond_{\nabla_{\diamond}}$.

Proof. (1) Let $\nabla$ be a finite quasi-modal operator on $\mathbf{A}$ and $a \in A$. By Lemma 2.8. we have

$$
\nabla_{\diamond_{\nabla}} a=\diamond_{\nabla}([a))=\nabla a .
$$

(2) Let $\diamond$ be a possibility modal operator on $\operatorname{Fi}_{\mathrm{f}}(\mathbf{A})$. First we prove that $\diamond_{\nabla_{\diamond}}([a))=\diamond([a))$, for every $a \in A$. By definition,

$$
\diamond_{\nabla_{\diamond}}([a))=\operatorname{Fig}\left(\bigcup\left\{\nabla_{\diamond} x: x \in[a)\right\}\right)=\operatorname{Fig}(\bigcup\{\diamond([x)): a \leq x\}) .
$$

Since $a \leq a$, it follows that $\diamond([a)) \subseteq \nabla_{\nabla_{\diamond}}([a))$. On the other hand, for each $a \leq x$, we have $[x) \subseteq[a)$ and $\diamond([x)) \subseteq \diamond([a))$. Thus, Fig $(\bigcup\{\diamond([x)): a \leq x\}) \subseteq \diamond([a))$. Hence, $\nabla_{\nabla_{\diamond}}([a))=\diamond([a))$, for all $a \in A$. Now, we show the general case. If $F \in \mathrm{Fi}_{\mathrm{f}}(A)$, then there exist $a_{1}, \ldots, a_{n} \in A$ such that $F=\left[a_{1}\right) \underline{\vee} \ldots \underline{\vee}\left[a_{n}\right)$. By Propositions 2.9 and 2.10 we have

$$
\begin{aligned}
\diamond_{\nabla_{\diamond}}(F) & =\diamond_{\nabla_{\diamond}}\left(\left[a_{1}\right) \underline{\vee} \ldots \underline{\vee}\left[a_{n}\right)\right) \\
& =\diamond_{\nabla_{\diamond}}\left(\left[a_{1}\right)\right) \underline{\vee} \ldots \underline{\vee} \nabla_{\nabla_{\diamond}}\left(\left[a_{n}\right)\right) \\
& =\diamond\left(\left[a_{1}\right)\right) \underline{\vee} \ldots \underline{\vee} \nabla\left(\left[a_{n}\right)\right) \\
& =\diamond\left(\left[a_{1}\right) \underline{\vee} \ldots \underline{\vee}\left[a_{n}\right)\right) \\
& =\diamond(F) .
\end{aligned}
$$

Then, by Theorem 2.11, we have that the finite quasi-modal operators on a distributive nearlattice $\mathbf{A}$ are in one to one correspondence with possibility modal operators on the distributive lattice of its finitely generated filters $\mathrm{Fi}_{\mathrm{f}}(\mathbf{A})$.

2.3. Representation for qm-distributive nearlattices. Our next objective is to give a representation theorem for qm-distributive nearlattices. Let $\langle\mathbf{A}, \nabla\rangle \in$ $\mathcal{D} \mathcal{N}_{\nabla}$. Let $R_{\nabla} \subseteq \mathrm{X}(A) \times \mathrm{X}(A)$ be the relation given by

$$
(P, Q) \in R_{\nabla} \Longleftrightarrow \gamma(P) \cap Q=\emptyset,
$$

and for each $F \in \operatorname{Fi}(A)$, define the set

$$
\beta(F)=\{P \in \mathrm{X}(A): P \cap F=\emptyset\} .
$$

If follows that $\beta(\gamma(P))=R_{\nabla}(P)$, for every $P \in \mathrm{X}(A)$. We consider the pair $\left\langle\mathrm{X}(A), R_{\nabla}\right\rangle$ and the operator $\square_{R_{\nabla}}$ of Example 2.4 given by $(*)$. 
Lemma 2.12. Let $\langle\mathbf{A}, \nabla\rangle \in \mathcal{D} \mathcal{N}_{\nabla}$. Then $\square_{R_{\nabla}}\left(\varphi_{A}(a)\right)=\beta(\nabla a)$, for every $a \in A$. Proof. Let $a \in A$ and $P \in \mathrm{X}(A)$. By Proposition 2.6. we have

$$
\begin{aligned}
P \notin \square_{R_{\nabla}}\left(\varphi_{A}(a)\right) & \Longleftrightarrow R_{\nabla}(P) \nsubseteq \varphi_{A}(a) \\
& \Longleftrightarrow \exists Q \in \mathrm{X}(A)[\gamma(P) \cap Q=\emptyset \text { and } a \in Q] \\
& \Longleftrightarrow a \notin \gamma(P) \\
& \Longleftrightarrow P \notin \beta(\nabla a) .
\end{aligned}
$$

So, $\square_{R_{\nabla}}\left(\varphi_{A}(a)\right)=\beta(\nabla a)$.

Definition 2.13. Let $\left\langle\mathbf{A}, \nabla_{1}\right\rangle,\left\langle\mathbf{B}, \nabla_{2}\right\rangle \in \mathcal{D} \mathcal{N}_{\nabla}$. We say that a map $h: A \rightarrow B$ is a qm-homomorphism if it satisfies the following conditions:

(1) $h$ is a homomorphism.

(2) $\operatorname{Fig}_{B}\left(h\left(\nabla_{1} a\right)\right)=\nabla_{2}(h(a))$, for every $a \in A$.

Moreover, if $h$ is an isomorphism we say that $h$ is a qm-isomorphism.

Lemma 2.14. Let $\left\langle\mathbf{A}, \nabla_{1}\right\rangle,\left\langle\mathbf{B}, \nabla_{2}\right\rangle,\left\langle\mathbf{C}, \nabla_{3}\right\rangle \in \mathcal{D N}_{\nabla}$. Let $h: A \rightarrow B$ and $g: B \rightarrow$ $C$ be two qm-homomorphisms. Then the composition $g \circ h$ is a qm-homomorphism.

Proof. Since $h: A \rightarrow B$ and $g: B \rightarrow C$ are two qm-homomorphisms, we have

$$
\operatorname{Fig}_{B}\left(h\left(\nabla_{1} a\right)\right)=\nabla_{2}(h(a)),
$$

for every $a \in A$, and

$$
\operatorname{Fig}_{C}\left(g\left(\nabla_{2} b\right)\right)=\nabla_{3}(g(b))
$$

for every $b \in B$. We prove that $\operatorname{Fig}_{C}\left((g \circ h)\left(\nabla_{1} a\right)\right)=\nabla_{3}((g \circ h)(a))$, for every $a \in A$. Note that

$$
\begin{aligned}
\nabla_{3}((g \circ h)(a)) & =\nabla_{3}(g(h(a))) \\
& =\operatorname{Fig}_{C}\left(g\left(\nabla_{2} h(a)\right)\right) \\
& =\operatorname{Fig}_{C}\left(g\left(\operatorname{Fig}_{B}\left(h\left(\nabla_{1} a\right)\right)\right)\right) .
\end{aligned}
$$

Since $h\left(\nabla_{1} a\right) \subseteq \operatorname{Fig}_{B}\left(h\left(\nabla_{1} a\right)\right)$, we have $g\left(h\left(\nabla_{1} a\right)\right) \subseteq g\left(\operatorname{Fig}_{B}\left(h\left(\nabla_{1} a\right)\right)\right)$. Then $\operatorname{Fig}_{C}\left(g\left(h\left(\nabla_{1} a\right)\right)\right) \subseteq \operatorname{Fig}_{C}\left(g\left(\operatorname{Fig}_{B}\left(h\left(\nabla_{1} a\right)\right)\right)\right)$, i.e., $\operatorname{Fig}_{C}\left((g \circ h)\left(\nabla_{1} a\right)\right) \subseteq \nabla_{3}((g \circ$ $h)(a))$. In order to prove the inverse inclusion, let us show that $g\left(\operatorname{Fig}_{B}\left(h\left(\nabla_{1} a\right)\right)\right) \subseteq$ $\operatorname{Fig}_{C}\left((g \circ h)\left(\nabla_{1} a\right)\right)$. If $y \in \operatorname{Fig}_{B}\left(h\left(\nabla_{1} a\right)\right)$, then there exist $y_{1}, \ldots, y_{n} \in\left[h\left(\nabla_{1} a\right)\right)$ such that $y_{1} \wedge \ldots \wedge y_{n}$ exists and $y=y_{1} \wedge \ldots \wedge y_{n}$. So, there are $x_{1}, \ldots, x_{n} \in \nabla_{1} a$ such that $h\left(x_{i}\right) \leq y_{i}$, for all $i \in\{1, \ldots, n\}$. Then $g(y)=g\left(y_{1}\right) \wedge \ldots \wedge g\left(y_{n}\right)$ and $(g \circ h)\left(x_{i}\right) \leq g\left(y_{i}\right)$, for all $i \in\{1, \ldots, n\}$. Hence, $g(y) \in \operatorname{Fig}_{C}\left((g \circ h)\left(\nabla_{1} a\right)\right)$ and the inclusion is valid. Thus,

$$
\operatorname{Fig}_{C}\left(g\left(\operatorname{Fig}_{B}\left(h\left(\nabla_{1} a\right)\right)\right)\right) \subseteq \operatorname{Fig}_{C}\left((g \circ h)\left(\nabla_{1} a\right)\right)
$$

and $\nabla_{3}\left((g \circ h)\left(\nabla_{1} a\right)\right) \subseteq \operatorname{Fig}_{C}\left((g \circ h)\left(\nabla_{1} a\right)\right)$. Therefore, $g \circ h$ is a qm-homomorphism.

By Lemma 2.14, we can conclude that we have the category whose objects are qm-distributive nearlattices and whose morphisms are qm-homomorphisms. 
Theorem 2.15. Let $\langle\mathbf{A}, \nabla\rangle \in \mathcal{D} \mathcal{N}_{\nabla}$. Then $\left\langle\varphi_{A}[\mathbf{A}], \nabla_{R_{\nabla}}\right\rangle$ is a qm-distributive nearlattice. Moreover, $\langle\mathbf{A}, \nabla\rangle$ and $\left\langle\varphi_{A}[\mathbf{A}], \nabla_{R_{\nabla}}\right\rangle$ are qm-isomorphic.

Proof. By Example 2.4 the structure $\left\langle\varphi_{A}[\mathbf{A}], \nabla_{R_{\nabla}}\right\rangle$ is a qm-distributive nearlattice. By Theorem 1.7 the map $\varphi_{A}: A \rightarrow \varphi_{A}[A]$ given by $\varphi_{A}(a)=\{P \in \mathrm{X}(A)$ : $a \notin P\}$ is an isomorphism. Then, it is easy to see that $\varphi_{A}(\nabla a) \in \operatorname{Fi}\left(\varphi_{A}[A]\right)$, for every $a \in A$. We have only to prove that $\varphi_{A}(\nabla a)=\nabla_{R_{\nabla}}\left(\varphi_{A}(a)\right)$. By Lemma 2.12 . Theorem 1.6, and Example 2.4 we have

$$
\begin{aligned}
\varphi_{A}(b) \notin \varphi_{A}(\nabla a) & \Longleftrightarrow b \notin \nabla a \\
& \Longleftrightarrow \exists Q \in \mathrm{X}(A)[\nabla a \cap Q=\emptyset \text { and } b \in Q] \\
& \Longleftrightarrow \exists Q \in \mathrm{X}(A)\left[Q \in \beta(\nabla a) \text { and } Q \notin \varphi_{A}(b)\right] \\
& \Longleftrightarrow \exists Q \in \mathrm{X}(A)\left[Q \in \square_{R_{\nabla}}\left(\varphi_{A}(a)\right) \text { and } Q \notin \varphi_{A}(b)\right] \\
& \Longleftrightarrow \square_{R_{\nabla}}\left(\varphi_{A}(a)\right) \nsubseteq \varphi_{A}(b) \\
& \Longleftrightarrow \varphi_{A}(b) \notin \nabla_{R_{\nabla}}\left(\varphi_{A}(a)\right) .
\end{aligned}
$$

Then, $\varphi_{A}(\nabla a)=\nabla_{R_{\nabla}}\left(\varphi_{A}(a)\right)$ and $\varphi_{A}$ is a qm-isomorphism.

By Theorem 2.15, we can identify the operator $\nabla$ with $\nabla_{R_{\nabla}}$ of Example 2.4.

\section{QuASI-MODAL CONGRUENCES}

In this section, we study a particular class of congruences compatible with quasimodal operators. Given a distributive nearlattice $\mathbf{A}$, let us denote by $\operatorname{Con}(\mathbf{A})=$ $\langle\operatorname{Con}(A), \vee, \wedge, \Delta, \Omega\rangle$ the bounded distributive lattice of congruences of $\mathbf{A}$.

Definition 3.1. Let $\langle\mathbf{A}, \nabla\rangle \in \mathcal{D N}_{\nabla}$ and $\theta \in \operatorname{Con}(A)$. We say that $\theta$ is a $q m$ congruence of $\mathbf{A}$ if for each $(a, b) \in \theta$ the following condition holds:

$$
\forall x \in \nabla a, \exists y \in \nabla b:(x, y) \in \theta .
$$

This condition together with symmetry of $\theta$ implies

$$
\forall x \in \nabla b, \exists y \in \nabla a:(x, y) \in \theta .
$$

We denote by $\operatorname{Con}_{\mathrm{qm}}(A)$ the set of all qm-congruences of $\langle\mathbf{A}, \nabla\rangle$.

Example 3.2. Let $\left\langle\mathbf{A}, \nabla_{1}\right\rangle,\left\langle\mathbf{B}, \nabla_{2}\right\rangle \in \mathcal{D} \mathcal{N}_{\nabla}$. If $h: A \rightarrow B$ is a qm-homomorphism, then $\operatorname{Ker} h \in \operatorname{Con}_{\mathrm{qm}}(A)$. Indeed, let $(a, b) \in \operatorname{Ker} h$ and $x \in \nabla_{1} a$. Then $h(x) \in$ $h\left(\nabla_{1} a\right) \subseteq \operatorname{Fig}_{B}\left(h\left(\nabla_{1} a\right)\right)$ and, since $h$ is a qm-homomorphism, $\operatorname{Fig}_{B}\left(h\left(\nabla_{1} a\right)\right)=$ $\nabla_{2}(h(a))$. So, $h(x) \in \nabla_{2}(h(b))=\operatorname{Fig}_{B}\left(h\left(\nabla_{1} b\right)\right)$, i.e., there exist $y_{1}, \ldots, y_{n} \in$ $\left[h\left(\nabla_{1} b\right)\right)$ such that $y_{1} \wedge \ldots \wedge y_{n}$ exists and $h(x)=y_{1} \wedge \ldots \wedge y_{n}$. It follows that there are $x_{1}, \ldots, x_{n} \in \nabla_{1} b$ such that $h\left(x_{i}\right) \leq y_{i}$, for all $i \in\{1, \ldots, n\}$. As $\nabla_{1} b$ is a filter, $m^{n-1}\left(x_{1}, \ldots, x_{n}, x\right) \in \nabla_{1} b$. On the other hand,

$$
\begin{aligned}
h\left(m^{n-1}\left(x_{1}, \ldots, x_{n}, x\right)\right) & =m^{n-1}\left(h\left(x_{1}\right), \ldots, h\left(x_{n}\right), h(x)\right) \\
& =\left[h\left(x_{1}\right) \vee h(x)\right] \wedge_{h(x)} \ldots \wedge_{h(x)}\left[h\left(x_{n}\right) \vee h(x)\right] \\
& \leq\left[y_{1} \vee h(x)\right] \wedge_{h(x)} \ldots \wedge_{h(x)}\left[y_{n} \vee h(x)\right] \\
& =\left[y_{1} \wedge \ldots \wedge y_{n}\right] \vee h(x) \\
& =h(x) .
\end{aligned}
$$


So, $h\left(m^{n-1}\left(x_{1}, \ldots, x_{n}, x\right)\right) \leq h(x)$. Since $h(x) \leq h\left(m^{n-1}\left(x_{1}, \ldots, x_{n}, x\right)\right)$ is always valid, we have $\left(x, m^{n-1}\left(x_{1}, \ldots, x_{n}, x\right)\right) \in \operatorname{Ker} h$. Therefore, Ker $h \in \operatorname{Con}_{\mathrm{qm}}(A)$.

Consider $\operatorname{Con}_{\mathrm{qm}}(\mathbf{A})=\left\langle\operatorname{Con}_{\mathrm{qm}}(A), \vee, \wedge, \Delta, \Omega\right\rangle$. We have the following result.

Lemma 3.3. Let $\langle\mathbf{A}, \nabla\rangle \in \mathcal{D} \mathcal{N}_{\nabla}$. Then $\operatorname{Con}_{\mathrm{qm}}(\mathbf{A})$ is a sublattice of $\operatorname{Con}(\mathbf{A})$.

Proof. Let $\theta_{1}, \theta_{2} \in \operatorname{Con}_{\mathrm{qm}}(A)$. We prove that $\theta_{1} \wedge \theta_{2}, \theta_{1} \vee \theta_{2} \in \operatorname{Con}_{\mathrm{qm}}(A)$. Let $(a, b) \in \theta_{1} \wedge \theta_{2}$ and $x \in \nabla a$. As $\theta_{1}$ and $\theta_{2}$ are qm-congruences, there exist $y, z \in \nabla b$ such that $(x, y) \in \theta_{1}$ and $(x, z) \in \theta_{2}$. Since $[x)$ is a bounded distributive lattice, there exist $(y \vee x) \wedge_{x}(z \vee x), x \wedge(y \vee x)$, and $x \wedge(z \vee x)$. Thus,

$$
\left(x \wedge(z \vee x),(y \vee x) \wedge_{x}(z \vee x)\right)=(x, m(y, z, x)) \in \theta_{1}
$$

and

$$
\left(x \wedge(y \vee x),(y \vee x) \wedge_{x}(z \vee x)\right)=(x, m(y, z, x)) \in \theta_{2},
$$

i.e., $(x, m(y, z, x)) \in \theta_{1} \wedge \theta_{2}$. Besides, since $\nabla b$ is a filter, we have $m(y, z, x) \in \nabla b$. It follows that $\theta_{1} \wedge \theta_{2} \in \operatorname{Con}_{\mathrm{qm}}(A)$.

Let $(a, b) \in \theta_{1} \vee \theta_{2}$ and $x \in \nabla a$. Then there exist $a=c_{0}, c_{1}, \ldots, c_{n}=b \in A$ such that $\left(c_{i}, c_{i+1}\right) \in \theta_{1} \cup \theta_{2}$, for all $i \in\{0, \ldots, n-1\}$. So, $x \in \nabla c_{0}$ and $\left(c_{0}, c_{1}\right) \in$ $\theta_{1} \cup \theta_{2}$. Since $\theta_{1}$ and $\theta_{2}$ are qm-congruences, there exists $y_{1} \in \nabla c_{1}$ such that $\left(x, y_{1}\right) \in \theta_{1} \cup \theta_{2}$. Then $y_{1} \in \nabla c_{1}$ and $\left(c_{1}, c_{2}\right) \in \theta_{1} \cup \theta_{2}$. Again, since $\theta_{1}$ and $\theta_{2}$ are qm-congruences, there exists $y_{2} \in \nabla c_{2}$ such that $\left(y_{1}, y_{2}\right) \in \theta_{1} \cup \theta_{2}$. By induction, there are $y_{1}, \ldots, y_{n} \in A$ such that $y_{j} \in \nabla c_{j}$, for all $j \in\{1, \ldots, n\}$, and $\left(x, y_{1}\right),\left(y_{k}, y_{k+1}\right) \in \theta_{1} \cup \theta_{2}$, for all $k \in\{1, \ldots, n-1\}$. Therefore, there is $y_{n} \in \nabla c_{n}=\nabla b$ such that $\left(x, y_{n}\right) \in \theta_{1} \vee \theta_{2}$. So, $\theta_{1} \vee \theta_{2}$ is a qm-congruence.

If $\langle\mathbf{A}, \nabla\rangle \in \mathcal{D} \mathcal{N}_{\nabla}$ and $\theta \in \operatorname{Con}_{\mathrm{qm}}(A)$, we define the quotient algebra as a pair $\left\langle\mathbf{A} / \theta, \nabla_{\theta}\right\rangle$ where $\mathbf{A} / \theta$ is the quotient distributive nearlattice of $\mathbf{A}$ by $\theta$ and $\nabla_{\theta}$ is defined as follows:

$$
\nabla_{\theta}[a]_{\theta}=\left\{[b]_{\theta}: b \in \nabla a\right\},
$$

for every $a \in A$. Since $\theta$ is a qm-congruence, the definition of $\nabla_{\theta}$ is independent of the choice of the representative of the equivalence class. Let $\pi_{\theta}: A \rightarrow A / \theta$ be the canonical homomorphism, i.e., $\pi_{\theta}(a)=[a]_{\theta}$. We have the following result.

Lemma 3.4. Let $\langle\mathbf{A}, \nabla\rangle \in \mathcal{D N}_{\nabla}$ and $\theta \in \operatorname{Con}(A)$. The following conditions are equivalent:

(1) $\theta \in \operatorname{Con}_{\mathrm{qm}}(A)$.

(2) $\operatorname{Fig}_{A / \theta}\left(\pi_{\theta}(\nabla a)\right)=\nabla_{\theta}\left(\pi_{\theta}(a)\right)$, for every $a \in A$.

Proof. (1) $\Rightarrow(2) \quad$ It is easy to see that $\nabla_{\theta}\left(\pi_{\theta}(a)\right) \subseteq \operatorname{Fig}_{A / \theta}\left(\pi_{\theta}(\nabla a)\right)$. We prove the other inclusion. Let $[b]_{\theta} \in \operatorname{Fig}_{A / \theta}\left(\pi_{\theta}(\nabla a)\right)$. Then there are $\left[x_{1}\right]_{\theta}, \ldots,\left[x_{n}\right]_{\theta} \in$ $\left[\pi_{\theta}(\nabla a)\right)$ such that $\left[x_{1}\right]_{\theta} \wedge \ldots \wedge\left[x_{n}\right]_{\theta}$ exists and $[b]_{\theta}=\left[x_{1}\right]_{\theta} \wedge \ldots \wedge\left[x_{n}\right]_{\theta}$. Thus, there exist $y_{1}, \ldots, y_{n} \in \nabla a$ such that $\left[y_{i}\right]_{\theta} \leq\left[x_{i}\right]_{\theta}$, for all $i \in\{1, \ldots, n\}$. We take the element $m^{n-1}\left(y_{1}, \ldots, y_{n}, b\right) \in A$. As $\nabla a$ is a filter, $m^{n-1}\left(y_{1}, \ldots, y_{n}, b\right) \in \nabla a$ 
and $\left[m^{n-1}\left(y_{1}, \ldots, y_{n}, b\right)\right]_{\theta} \in \nabla_{\theta}\left(\pi_{\theta}(a)\right)$. It follows that

$$
\begin{aligned}
{\left[m^{n-1}\left(y_{1}, \ldots, y_{n}, b\right)\right]_{\theta} } & =\left(\left[y_{1}\right]_{\theta} \vee[b]_{\theta}\right) \wedge_{[b]_{\theta}} \ldots \wedge_{[b]_{\theta}}\left(\left[y_{n}\right]_{\theta} \vee[b]_{\theta}\right) \\
& \leq\left(\left[x_{1}\right]_{\theta} \vee[b]_{\theta}\right) \wedge_{[b]_{\theta}} \cdots \wedge_{[b]_{\theta}}\left(\left[x_{n}\right]_{\theta} \vee[b]_{\theta}\right) \\
& =\left(\left[x_{1}\right]_{\theta} \wedge \ldots \wedge\left[x_{n}\right]_{\theta}\right) \vee[b]_{\theta} \\
& =[b]_{\theta}
\end{aligned}
$$

Then $\left[m^{n-1}\left(y_{1}, \ldots, y_{n}, b\right)\right]_{\theta} \leq[b]_{\theta}$. On the other hand, by the monotonicity of $\pi_{\theta}$ it follows that $[b]_{\theta} \leq\left[m^{n-1}\left(y_{1}, \ldots, y_{n}, b\right)\right]_{\theta}$. Thus, $[b]_{\theta}=\left[m^{n-1}\left(y_{1}, \ldots, y_{n}, b\right)\right]_{\theta}$ and $[b]_{\theta} \in \nabla_{\theta}\left(\pi_{\theta}(a)\right)$. We conclude that $\operatorname{Fig}_{A / \theta}\left(\pi_{\theta}(\nabla a)\right)=\nabla_{\theta}\left(\pi_{\theta}(a)\right)$.

$(2) \Rightarrow(1) \quad$ Let $a, b \in A$ be such that $(a, b) \in \theta$ and $x \in \nabla a$. Then $[x]_{\theta} \in$ $\pi_{\theta}(\nabla a) \subseteq \operatorname{Fig}_{A / \theta}\left(\pi_{\theta}(\nabla a)\right)$. Since $\operatorname{Fig}_{A / \theta}\left(\pi_{\theta}(\nabla a)\right)=\nabla_{\theta}\left(\pi_{\theta}(a)\right)$ and $\pi_{\theta}(a)=\pi_{\theta}(b)$, we have $[x]_{\theta} \in \nabla_{\theta}\left(\pi_{\theta}(b)\right)=\operatorname{Fig}_{A / \theta}\left(\pi_{\theta}(\nabla b)\right)$, i.e., $[x]_{\theta} \in \operatorname{Fig}_{A / \theta}\left(\pi_{\theta}(\nabla b)\right)$. Thus, there exist $\left[x_{1}\right]_{\theta}, \ldots,\left[x_{n}\right]_{\theta} \in\left[\pi_{\theta}(\nabla b)\right)$ such that $\left[x_{1}\right]_{\theta} \wedge \ldots \wedge\left[x_{n}\right]_{\theta}$ exists and $[x]_{\theta}=$ $\left[x_{1}\right]_{\theta} \wedge \ldots \wedge\left[x_{n}\right]_{\theta}$. As $\pi_{\theta}: A \rightarrow A / \theta$ is an onto homomorphism and $\nabla b$ is a filter, it follows that $\pi_{\theta}(\nabla b) \in \mathrm{Fi}(A / \theta)$ and $\left[\pi_{\theta}(\nabla b)\right)=\pi_{\theta}(\nabla b)$. So, $\left[x_{1}\right]_{\theta}, \ldots,\left[x_{n}\right]_{\theta} \in$ $\pi_{\theta}(\nabla b)$ and $[x]_{\theta}=\left[x_{1}\right]_{\theta} \wedge \ldots \wedge\left[x_{n}\right]_{\theta} \in \pi_{\theta}(\nabla b)$. Then, there is $y \in \nabla b$ such that $[x]_{\theta}=[y]_{\theta}$ and $(x, y) \in \theta$. Therefore, $\theta$ is a qm-congruence.

Theorem 3.5. Let $\langle\mathbf{A}, \nabla\rangle \in \mathcal{D} \mathcal{N}_{\nabla}$ and $\theta \in \operatorname{Con}_{\mathrm{qm}}(A)$. Then $\left\langle\mathbf{A} / \theta, \nabla_{\theta}\right\rangle$ is a $q m$-distributive nearlattice and $\pi_{\theta}$ a qm-homomorphism from $\mathbf{A}$ onto $\mathbf{A} / \theta$.

Proof. The result follows by Lemma 3.4

Theorem 3.6. Let $\left\langle\mathbf{A}, \nabla_{1}\right\rangle,\left\langle\mathbf{B}, \nabla_{2}\right\rangle \in \mathcal{D N}_{\nabla}$. Let $h: A \rightarrow B$ be an onto qmhomomorphism. Then there exists a qm-isomorphism $f: A / \operatorname{Ker} h \rightarrow B$ such that $h=f \circ \pi_{K}$.

Proof. By Example 3.2 Ker $h$ is a qm-congruence. Then $\left\langle\mathbf{A} / \operatorname{Ker} h, \nabla_{K}\right\rangle$ is a qmdistributive nearlattice. By results of universal algebra (see [4]), there exists an isomorphism $f: A / \operatorname{Ker} h \rightarrow B$ such that $h=f \circ \pi_{K}$. We prove that $f$ is a qmhomomorphism, i.e.,

$$
\operatorname{Fig}_{B}\left(f\left(\nabla_{K}[a]_{K}\right)\right)=\nabla_{2}\left(f\left([a]_{K}\right)\right),
$$

for every $[a]_{K} \in A / \operatorname{Ker} h$. Since $\nabla_{K}[a]_{K}$ is a filter of $A / \operatorname{Ker} h$ and $f$ is an isomorphism, we have that $f\left(\nabla_{K}[a]_{K}\right)$ is a filter of $B$. We show that $f\left(\nabla_{K}[a]_{K}\right)=$ $\nabla_{2}\left(f\left([a]_{K}\right)\right)$. Using that $\pi_{K}$ is a qm-homomorphism and that $\pi K\left(\nabla_{1}(a)\right)$ is a filter of $A / \operatorname{Ker} h$, we have

$$
\begin{aligned}
f\left(\nabla_{K}[a]_{K}\right) & =f\left(\nabla_{K} \pi_{K}(a)\right)=f\left(\operatorname{Fig}_{A / \operatorname{Ker} h}\left(\pi_{K}\left(\nabla_{1} a\right)\right)\right) \\
& =f\left(\pi_{K}\left(\nabla_{1} a\right)\right)=h\left(\nabla_{1} a\right) \\
& =\operatorname{Fig}_{B}\left(h\left(\nabla_{1} a\right)\right)=\nabla_{2}(h(a)) \\
& =\nabla_{2}\left(f\left(\pi_{K}(a)\right)\right)=\nabla_{2}\left(f\left([a]_{K}\right)\right) .
\end{aligned}
$$

Then $f$ is a qm-isomorphism from $\mathbf{A} / \operatorname{Ker} h$ on $\mathbf{B}$. 
Let $\mathbf{A}$ be a distributive nearlattice and define the map $f: \operatorname{Con}\left(\mathrm{Fi}_{\mathrm{f}}(A)\right) \rightarrow$ $\operatorname{Con}(A)$ via

$$
(a, b) \in f(\theta) \Longleftrightarrow([a),[b)) \in \theta,
$$

for every $\theta \in \operatorname{Con}\left(\mathrm{Fi}_{\mathrm{f}}(A)\right)$. In [17] (see also [13]), the authors show that $f$ is an isomorphism. We extend this result to the class of finite quasi-modal distributive nearlattices.

Theorem 3.7. Let A be a distributive nearlattice. Let $\diamond$ be a possibility modal operator on $\mathrm{Fi}_{\mathrm{f}}(\mathbf{A})$. Then $f: \operatorname{Con}\left(\left\langle\mathrm{Fi}_{\mathrm{f}}(A), \diamond\right\rangle\right) \rightarrow \operatorname{Con}_{\mathrm{qm}}\left(\left\langle A, \nabla_{\diamond}\right\rangle\right)$ given by $\odot$ is an isomorphism.

Proof. We prove that $f$ is well-defined and $f^{-1}(\psi) \in \operatorname{Con}\left(\left\langle\operatorname{Fi}_{\mathrm{f}}(A), \diamond\right\rangle\right)$, for every $\psi \in \mathrm{Con}_{\mathrm{qm}}\left(\left\langle A, \nabla_{\diamond}\right\rangle\right)$.

Let $\theta \in \operatorname{Con}\left(\left\langle\operatorname{Fi}_{\mathrm{f}}(A), \diamond\right\rangle\right)$. Let $(a, b) \in f(\theta)$ and $x \in \nabla_{\diamond} a=\diamond([a))$. Then $([a),[b)) \in \theta$ and $(\diamond([a)), \diamond([b))) \in \theta$. Since $\diamond([a)), \diamond([b)) \in \mathrm{Fi}_{\mathrm{f}}(A)$, there exist $a_{1}, \ldots, a_{n}, b_{1}, \ldots, b_{k} \in A$ such that $\diamond([a))=\left[a_{1}\right) \underline{\vee} \ldots \underline{\vee}\left[a_{n}\right)$ and $\diamond([b))=\left[b_{1}\right) \underline{\vee}$ $\ldots \underline{\vee}\left[b_{k}\right)$. So, $\left(\left[a_{1}\right) \underline{\vee} \ldots \underline{\vee}\left[a_{n}\right),\left[b_{1}\right) \underline{\vee} \ldots \underline{\vee}\left[b_{k}\right)\right) \in \theta$. As $[x] \subseteq\left[a_{1}\right) \underline{\vee} \ldots \underline{\vee}\left[a_{n}\right)$, we have

$$
\begin{aligned}
\left([x),[x) \cap\left(\left[b_{1}\right) \underline{\vee} \ldots \underline{\vee}\left[b_{k}\right)\right)\right) & =\left([x),\left[\left(b_{1} \vee x\right) \wedge \ldots \wedge\left(b_{k} \vee x\right)\right)\right) \\
& =\left([x),\left[m^{k-1}\left(b_{1}, \ldots, b_{k}, x\right)\right)\right) \in \theta,
\end{aligned}
$$

where $m^{k-1}\left(b_{1}, \ldots, b_{k}, x\right) \in \diamond([b))$. Therefore, $f$ is well-defined.

Let $\psi \in \operatorname{Con}_{\mathrm{qm}}\left(\left\langle A, \nabla_{\diamond}\right\rangle\right)$ and let $\theta \in \operatorname{Con}\left(\mathrm{Fi}_{\mathrm{f}}(A)\right)$ be such that $f(\theta)=\psi$. We prove that $\theta \in \operatorname{Con}\left(\left\langle\operatorname{Fi}_{\mathrm{f}}(A), \diamond\right\rangle\right)$. First, we show that if $([a),[b)) \in \theta$, then $(\diamond([a)), \diamond([b))) \in \theta$, for every $a, b \in A$. If $([a),[b)) \in \theta$, then by definition of $f$ we have $(a, b) \in \psi$. Since $\diamond([a)), \diamond([b)) \in \mathrm{Fi}_{\mathrm{f}}(A)$, there exist $a_{1}, \ldots, a_{n}, b_{1}, \ldots, b_{k} \in A$ such that $\diamond([a))=\left[a_{1}\right) \underline{\vee} \ldots \underline{\vee}\left[a_{n}\right)$ and $\diamond([b))=\left[b_{1}\right) \underline{\vee} \ldots \underline{\vee}\left[b_{k}\right)$. As $\psi$ is a qmcongruence, $(a, b) \in \psi$ and $a_{i} \in \diamond([a))=\nabla_{\diamond} a$, for all $i \in\{1, \ldots, n\}$. It follows that there are $y_{1}, \ldots, y_{n} \in A$ such that $y_{i} \in \nabla_{\diamond} b=\diamond([b))$ and $\left(a_{i}, y_{i}\right) \in \psi=f(\theta)$, i.e., $\left(\left[a_{i}\right),\left[y_{i}\right)\right) \in \theta$, for all $i \in\{1, \ldots, n\}$. Then

$$
\left(\left[a_{1}\right) \underline{\vee} \ldots \underline{\vee}\left[a_{n}\right),\left[y_{1}\right) \underline{\vee} \ldots \underline{\vee}\left[y_{n}\right)\right)=\left(\diamond([a)),\left[y_{1}\right) \underline{\vee} \ldots \underline{\vee}\left[y_{n}\right)\right) \in \theta .
$$

On the other hand, as $y_{i} \in \diamond([b))$ for all $i \in\{1, \ldots, n\},\left[y_{1}\right) \underline{\vee} \ldots \underline{\vee}\left[y_{n}\right) \subseteq \diamond([b))$ and $\left(\diamond([a)) \cap \diamond([b)),\left[y_{1}\right) \underline{\vee} \ldots \underline{\vee}\left[y_{n}\right)\right) \in \theta$. Thus, by transivity of $\theta$, we have $(\diamond([a)), \diamond([a)) \cap \diamond([b))) \in \theta$. Analogously, we can prove that $(\diamond([b)), \diamond([a)) \cap$ $\diamond([b))) \in \theta$. Hence, $(\diamond([a)), \diamond([b))) \in \theta$.

Now, we prove the general case. Let $F, G \in \mathrm{Fi}_{\mathrm{f}}(A)$ be such that $(F, G) \in \theta$. Then there exist $a_{1}, \ldots, a_{n}, b_{1}, \ldots, b_{k} \in A$ such that $F=\left[a_{1}\right) \underline{\vee} \ldots \underline{\vee}\left[a_{n}\right)$ and $G=\left[b_{1}\right) \underline{\vee} \ldots \underline{\vee}\left[b_{k}\right)$. So, $\left(\left[a_{i}\right), G \cap\left[a_{i}\right)\right) \in \theta$, for all $i \in\{1, \ldots, n\}$. Note that

$$
\begin{aligned}
G \cap\left[a_{i}\right) & =\left(\left[b_{1}\right) \underline{\vee} \ldots \underline{\vee}\left[b_{k}\right)\right) \cap\left[a_{i}\right) \\
& =\left[\left(b_{1} \vee a_{i}\right) \wedge_{a_{i}} \ldots \wedge_{a_{i}}\left(b_{k} \vee a_{i}\right)\right) \\
& =\left[m^{k-1}\left(b_{1}, \ldots, b_{k}, a_{i}\right)\right) .
\end{aligned}
$$


Thus, $\left(\left[a_{i}\right),\left[m^{k-1}\left(b_{1}, \ldots, b_{k}, a_{i}\right)\right)\right) \in \theta$ and $\left(\diamond\left(\left[a_{i}\right)\right), \diamond\left(\left[m^{k-1}\left(b_{1}, \ldots, b_{k}, a_{i}\right)\right)\right)\right) \in \theta$, for all $i \in\{1, \ldots, n\}$. It follows that

$\left(\diamond\left(\left[a_{1}\right)\right) \underline{\vee} \ldots \underline{\vee} \diamond\left(\left[a_{n}\right)\right), \diamond\left(\left[m^{k-1}\left(b_{1}, \ldots, b_{k}, a_{1}\right)\right)\right) \underline{\vee} \ldots \underline{\vee} \diamond\left(\left[m^{k-1}\left(b_{1}, \ldots, b_{k}, a_{n}\right)\right)\right)\right) \in \theta$.

Since $\diamond\left(\left[a_{1}\right)\right) \underline{\vee} \ldots \underline{\vee} \nabla\left(\left[a_{n}\right)\right)=\diamond\left(\left[a_{1}\right) \underline{\vee} \ldots \underline{\vee}\left[a_{n}\right)\right)=\diamond(F)$ and

$$
\begin{aligned}
& \diamond\left(\left[m^{k-1}(\right.\right.\left.\left.\left.b_{1}, \ldots, b_{k}, a_{1}\right)\right)\right) \underline{\vee} \ldots \underline{\vee} \diamond\left(\left[m^{k-1}\left(b_{1}, \ldots, b_{k}, a_{n}\right)\right)\right) \\
& \quad=\diamond\left(G \cap\left[a_{1}\right)\right) \underline{\vee} \ldots \underline{\vee} \nabla\left(G \cap\left[a_{n}\right)\right)=\diamond\left(\left(G \cap\left[a_{1}\right)\right) \underline{\vee} \ldots \underline{\vee}\left(G \cap\left[a_{n}\right)\right)\right) \\
& \quad=\diamond\left(G \cap\left(\left[a_{1}\right) \underline{\vee} \ldots \underline{\vee}\left[a_{n}\right)\right)\right)=\diamond(G \cap F),
\end{aligned}
$$

we have $(\diamond(F), \diamond(G \cap F)) \in \theta$. Similarly, we can show that $(\diamond(G), \diamond(G \cap F)) \in \theta$. Therefore, $\theta \in \operatorname{Con}\left(\left\langle\operatorname{Fi}_{\mathrm{f}}(A), \diamond\right\rangle\right)$ and $f$ is an isomorphism.

\section{REFERENCES}

[1] J. C. Abbott, Semi-boolean algebra, Mat. Vesnik 4(19) (1967), 177-198. MR 0239957

[2] J. C. Abbott, Implicational algebras, Bull. Math. Soc. Sci. Math. R. S. Roumanie 11(59) (1967), no. 1, 3-23. MR 0239958.

[3] J. Araújo and M. Kinyon, Independent axiom systems for nearlattices, Czechoslovak Math. J. 61(136) (2011), no. 4, 975-992. MR 2886250

[4] S. Burris and H. P. Sankappanavar, A course in universal algebra, Graduate Texts in Mathematics, 78, Springer-Verlag, New York, 1981. MR 0648287

[5] S. Celani, Quasi-modal algebras, Math. Bohem. 126 (2001), no. 4, 721-736. MR 1869464

[6] S. Celani, Subdirectly irreducible quasi-modal algebras, Acta Math. Univ. Comenian. (N.S.) 74 (2005), no. 2, 219-228. MR 2195481

[7] S. Celani, Modal Tarski algebras, Rep. Math. Logic 39 (2005), 113-126. MR 2207379

[8] S. Celani and I. Calomino, Stone style duality for distributive nearlattices, Algebra Universalis 71 (2014), no. 2, 127-153. MR 3183387

[9] S. Celani and I. Calomino, On homomorphic images and the free distributive lattice extension of a distributive nearlattice, Rep. Math. Logic 51 (2016), 57-73. MR 3562693

[10] S. Celani and I. Calomino, Distributive nearlattices with a necessity modal operator, Math. Slovaca 69 (2019), no. 1, 35-52. MR 3903625

[11] A. Chagrov and M. Zakharyaschev, Modal logic, Oxford Logic Guides, 35, The Clarendon Press, Oxford University Press, New York, 1997. MR 1464942

[12] I. Chajda and R. Halaš, An example of a congruence distributive variety having no nearunanimity term, Acta Univ. M. Belii Ser. Math. 13 (2006), 29-31. MR 2353310

[13] I. Chajda, R. Halaš and J. Kühr, Semilattice structures, Research and Exposition in Mathematics, 30, Heldermann Verlag, Lemgo, 2007. MR 2326262

[14] I. Chajda and M. Kolař́k, Ideals, congruences and annihilators on nearlattices, Acta Univ. Palack. Olomuc. Fac. Rerum Natur. Math. 46 (2007), 25-33. MR 2387490

[15] I. Chajda and M. Kolař́ḱ, Nearlattices, Discrete Math. 308 (2008), no. 21, 4906-4913. MR 2446101

[16] R. Cignoli, Quantifiers on distributive lattices, Discrete Math. 96 (1991), no. 3, $183-197$. MR 1139446

[17] W. H. Cornish and R. C. Hickman, Weakly distributive semilattices, Acta Math. Acad. Sci. Hungar. 32 (1978), no. 1-2, 5-16. MR 0551490. 
[18] L. J. González, The logic of distributive nearlattices, Soft Comput. 22 (2018), no. 9, 27972807. https://doi.org/10.1007/s00500-017-2750-0

[19] L. J. González, Selfextensional logics with a distributive nearlattice term, Arch. Math. Logic 58 (2019), no. 1-2, 219-243. MR 3902813

[20] R. Halaš, Subdirectly irreducible distributive nearlattices, Miskolc Math. Notes 7 (2006), no. 2, 141-146 (2007). MR 2310273

[21] R. Hickman, Join algebras, Comm. Algebra 8 (1980), no. 17, 1653-1685. MR 0585925

[22] A. Petrovich, Distributive lattices with an operator, Studia Logica 56 (1996), no. 1-2, 205224. MR 1382174

Ismael Calomino $\bowtie$

CIC and Departamento de Matemática, Facultad de Ciencias Exactas, Universidad Nacional del Centro, Pinto 399, 7000 Tandil, Argentina

calomino@exa.unicen.edu.ar

Sergio A. Celani

CONICET and Departamento de Matemática, Facultad de Ciencias Exactas, Universidad

Nacional del Centro, Pinto 399, 7000 Tandil, Argentina

celani@exa.unicen.edu.ar

Luciano J. González

Universidad Nacional de La Pampa. Facultad de Ciencias Exactas y Naturales. Santa Rosa, Argentina

lucianogonzalez@exactas.unlpam.edu.ar

Received: March 27, 2019

Accepted: September 28, 2019 